\title{
Efficient Opportunistic Overlay Prototype with Reliable Routing and Distributed Resource Discovery Services
}

\author{
Mr. Madhav Gopal Sharma \\ Research Scholar, Department of Computer Science \\ \& IT, Singhania University, Rajasthan
}

\author{
Prof. (Dr.) R. B. Patel \\ Professor, Department of Computer Science \& \\ Engineering, Chandigarh College of Engineering and \\ Technology, Chandigarh, India
}

\begin{abstract}
Now are days wireless communication system are growing in various public as well as private sectors to perform different services meanwhile automatic execution of various data and voice communication services, security and quality enhancement will be the major issues during P2P distributed services such scenario demands more transparent and powerful framework to achieve P2P networking goals and services. When we talk about such a distributed P2P network we always face, some critical issues regarding P2P routing, distributed resource sharing, security apart from this we can experience in many real-time applications, movable devices are critical to track and access during operation and services, it needs some requirement to enhance the distributed P2P services regarding file transfer and their replication as per the demand of distributed server over the network P2P security enhancement imparting the research for real big challenge .
\end{abstract}

Routing is critical and essential issue in distributed resource sharing, in this paper we present a routing protocol that support distributed networking services as well as routing efficiency it is named as RRS protocol it works based on four major aspects like resource node distance, resource angle, the energy level of the resources and the bandwidth link. The proposed policy also improved the quality of service specifications like reliability and stability with reduced energy consumption. In next section we show the algorithmic model along with the benefits of RRS protocols along with its implementation, in last session we explain the simulation and implementation level detail that define how this technology is beneficial for future networking trends and the comparative analysis will put proposed protocol valuable place as it defines it is more efficient and effective protocol.

Keywords: Distributed Resource sharing, P2P, Reliability, Stability Quality of services.

\section{INTRODUCTION}

The RRS routing protocol is very efficient routing protocol. Here, in this we tried to develop a new algorithm to utilize resource, place the resources and move the resources in the network. It helps to transfer the packets with minimum number of hops, less energy consumption and dynamic bandwidth utilization. On the basis of the consideration, we designed the RRS network is as follows:

\section{Step I: Place the resources:}

The first step of RRS protocol is to give a correct place for each resource. Suppose, the resource area is $1000 \mathrm{~cm} \times 1000 \mathrm{~cm}$ and number of resources are 10.The all resources are plotted within this area.

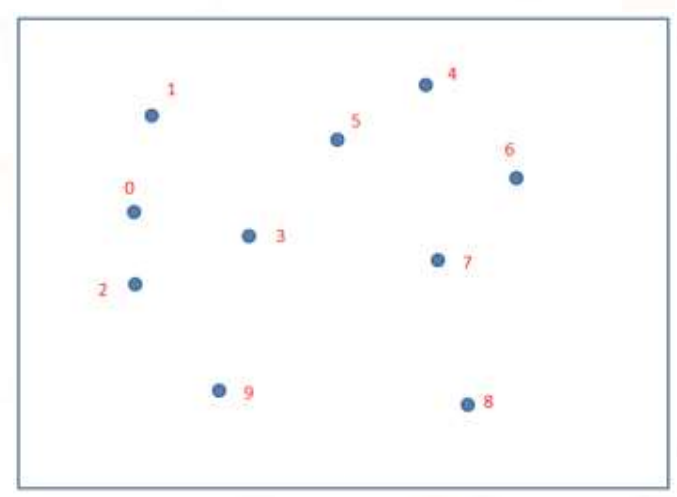

Figure 1.1: The Resource Area

The above Figure1.1 explains that all the ten resources are placed between the axis (within the $\mathrm{X}$ axis and $\mathrm{Y}$ axis). All the resources in the network are placed at different distance, different angle, and having different energy and bandwidth.

\section{Step II: Find the source and destination:}

The RRS protocol finds the source node and the destination resource. i.e. the way of transfer data from one resource to another resource. If the data transfer from $0^{\text {th }}$ resource to $6^{\text {th }}$ resource means, RRS consider the distance, angle, energy and bandwidth to find a 
correct path to transfer data from $0^{\text {th }}$ resource to $6^{\text {th }}$ resource.

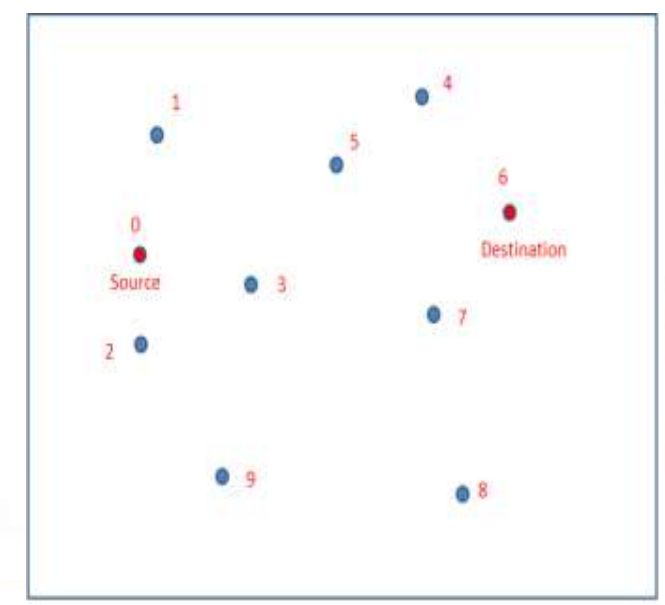

Figure 1.2: Source and Destination nodes.

\section{Step III: Find the distance between resources:}

For finding the distances of each resource from the place of source with the help of RRS protocol, we maintain the following table. First of all we determine the distance from the source place of $0^{\text {th }}$ resource.

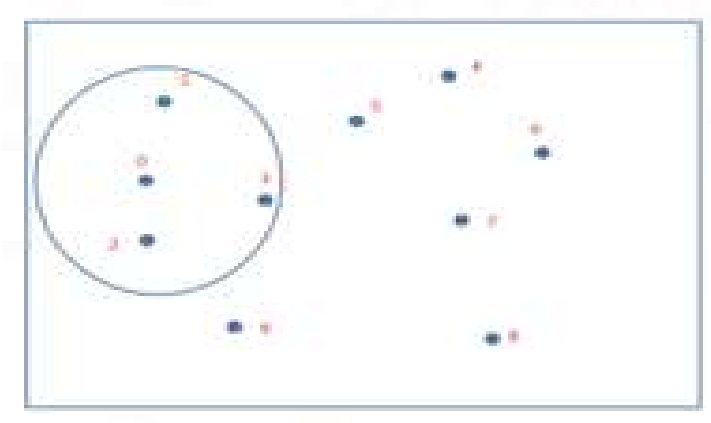

Figure 1.3: The Neighbor Resource of $0^{\text {th }}$ Resource

\section{LITERATURE SURVEY}

QoS is one of the major issue ever one talk about the reliable and efficient services because services for voice and data is compulsory to follow the aspect covered in the term like QoS that suppose to get many things like reliability, security, confidentiality, integrity and manageability during the complete flow of communication, that provides congestion less network communication architecture in [14]. Many mechanism has been proposed previously they discussed service to improve best effort flows but all are not sufficient and suitable for the point of view of quality of service requirements, proposed scheme provides such quality of services with the help of equation based mathematical construct to get congestion control for different network working scenario, algorithm support congestion control mechanism with QoS service architecture, method consist functions like data loss control at router end, $\mathrm{p} 2 \mathrm{p}$ resource sharing problem control at sender and receiver end [16].

In [17] author proposed a new $\mathrm{p} 2 \mathrm{p}$ mechanism with adaptive congestion window, designed to improve the performance of traditional TCP Reno cause problem during decline of connection protocol due to the over flow of bottleneck link buffer affect the performance of overall algorithm used for the increasing traffic scenario. Proposed network scheme define a modified in already defined TCP algorithm based on the distribution of available link capacity, such capacity has been distributed in some intelligent share propositions. Proposed ideal analyze the limitation of traditional TCP protocol and define new TCP mechanism called as ABE_TCP which has the ability to dynamically manage the congestion window at run time for wireless networking environment that works on the calculation of available bandwidth. Proposed algorithm getting decision to adjust congestion window on run time and adjust the slow start threshold dynamically with the help of parameter adjustment and setting scenario that result the degree of packet loss due to overflow error is getting less and increases performance effectively [20].

[18] proposed a impressive novel approach named Performance Analysis of RED with Difference TCP peer to peer resource sharing Mechanism, it is special designed for Internet, internet is growing technology at today's environment, so that traffic control is one of the issue of discussion, that motivates the researchers to resolve the problem with performance modeling, the proposed scheme presents a stochastic queuing model to control congestion and improve performance, scheme consist a new analytical model that works integrated with the collaboration of tradition RED model, model works on the basic principal of reduction of traffic load when channels get long delay, where packet has been going drop as per the need of the network scenario, algorithm perform evaluation of queue memory optimization , the system describes the performance valuation from different point of view like mean system occupancy and delay, evaluation of data loss and throughput has 
been considered by the system as a packet dropping model [19] .

\section{RELATED WORK}

In present communication technology either it is wire or wireless perform routing using peer to peer distributed network does unique challenges. Developers of routing protocols for peer to peer network need to address several issues. In this chapter author discuses the problem issue of $\mathrm{p} 2 \mathrm{p}$ distributed network and the routing protocols available for Peer to peer network along with the working framework of some protocols like DSDV, DSR, AODV, etc. apart from this author also analyzed the benefits and drawback of traditional protocols so that at the end of the paper author can compare performance and routing issues of proposed model with previous one. This chapter concludes with a summary of routing in peer to peer network.

\section{TRADITIONAL ROUTING PROTOCOLS}

Traditionally we can classify the routing protocols in three major categories like Proactive, Hybrid and on demand routing protocols. As in describes with the help of Figure 2.1 shows the classification of the routing protocols for traditional implemented and used in P2P network.

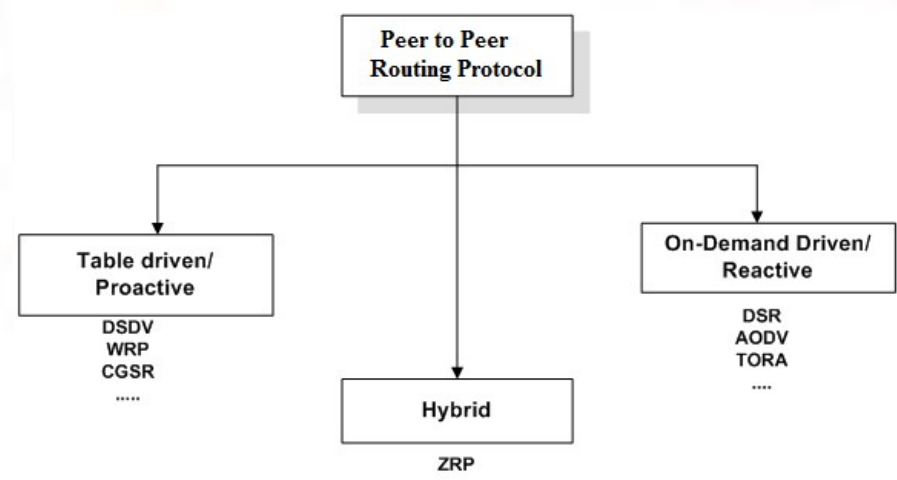

Figure 2.1: Classification of peer to peer network routing protocols

\section{A. Table-driven/Proactive Routing Protocols}

In this category, the protocols will perform routing in the way where each participated node will manage their individual information table, which has been shared with the other node of the network periodically. In this policy information are periodically upgraded by the way to board cast routing information to the next nearest node so that one can say it is the extension of traditional RIP protocol [1], here we have few examples which comes under into the category of Proactive routing protocols like DSDV as in [3], WRP used for wireless routing protocol, another can be CGSR works on the mechanism of cluster based routing scheme as in [5], in coming section we are going to discuss this protocols in brief

\section{B. Destination Sequenced Distance Vector (DSDV)}

This another protocol called DSDV, which works on the concept of distributed Bellman Ford strategy, in this technique as discussed in [6], ever node will manage neighbor node detail and distance value to perform efficient routing The Destination Sequenced Distance Vector (DSDV) protocol is works with multiple nodes, which consist sequencing by sequence no in order to achieve nodes data integrity, such practice will avoid looping in routing as in [2], in case if router found two same sequence no, so that the one with different distance value of route metric will got selected, to get route refresh and updating has to be performed on specific time period, in this way all the network node will multicast their addressing detail to remaining nodes of the network.

\section{Wireless Routing Protocol (WRP)}

As described in [4], The Wireless Routing protocol (WRP) comes under the category of proactive protocols performs similar as we discussed DSDV in last section. The major difference between these two protocols is the management of routing information table, as we know DSDV will not maintain table at every node but in WRP information table should be managed by every participated node as describes by following way.

Routing table (RT): It is essential to manage the routing details such information will be maintain at routing table having related information to destination node, it can be identified by predecessor, successor and interface address to get accuracy in routing.

Link Cost Table (LCT): This table manages the cost measurement belongs to different path and related destination, the objective of this table is to manage least cost computation to perform shortest path routing. 
Distance Table (DT): It is the only table used in this process which maintains destination node details.

Message Retransmission List (MRL): Massage management is necessary part of communication; this table contains details regarding message lost, retransmission, and message updating and message delivery time details. It is also manage acknowledgement detail with the help of flag.

\section{On-Demand/Reactive Routing Protocols}

Protocols are used based on user demand for routing process that's why we also called it as on demand routing protocol, in this approach two common things are Routing Invention and Path maintenance. In route invention process it will intimate to the related route address which is already available in its cache so that nodes who want to communicate with other then destination rely against request node. In the nest process route maintenance table will manage broken link details and acknowledgments details.

\section{E. Dynamic Source Routing (DSR) Protocol}

In[8], the DSR works on the concept of on demand source routing scheme, it is one of the major protocol of on demand category, in source routing, every node will able to traversal the packet to the destination node based on the information available in the routing table, so that as user demand for transferring the data to any specific node they no need to broadcast the request first but it will invent the next to next and more sequential route to proceed the request on demand services as soon as possible.

\section{F. Ad hoc On-demand Distance Vector (AODV)}

As describes in [9] the AODV is the most traditional protocol used regarding routing for $\mathrm{Ad}$ hoc networking AODV is good enough compare to lastly discussed protocols since it is the combination of DSDV and DSR so that AODV uses a on demand policy to finding path and it uses table driven policy to determine the most suitable route, specially it uses destination sequence number to perform data and node integrity.

\section{G. Hybrid Routing Protocols}

This category will be invented after proactive and reactive scheme so that it uses the concept of both in different aspect, now it is the combination of on demand and proactive routing protocols; the major objective of this protocol is to minimize the overheads of table driven and on demand services. To understand the working policy of Hybrid protocol we will go through with Zone Routing Protocol (ZRP) as an example of Hybrid protocols.

\section{H. Zone Routing Protocol (ZRP)}

ZRP perform routing using best service features of both reactive and on demand routing protocols services, the working frame work of this policy works on following four components : (i) Intra Zone Routing Protocol (IARP) (ii) Reactive Inter zone Routing Protocol (IERP), and (iii) Border cast Resolution Protocol (BRP) as in [10].

In this protocols routing scheme the complete network has been distributed in multiple zones, such zone represent different type of network it may be wired, wireless or the combination of the both types. Where IARP used to perform routing within the zone range uses table driven routing. In this case if any node belongs to two different zone like as in figure node A. so it is possible that a node can exchange his information periodically between multiple zones in this case routing has been done by IERP, IERP allow a node to communication with another node available at the border position of zone, $y$ broadcasting the packets for routing information exchange. For example, in Figure 2.2 1illustrate the same scenario where node 2 wand communicate with node 7 , so that zoning will works and it will send request packets to nodes 1,3 and 5 .

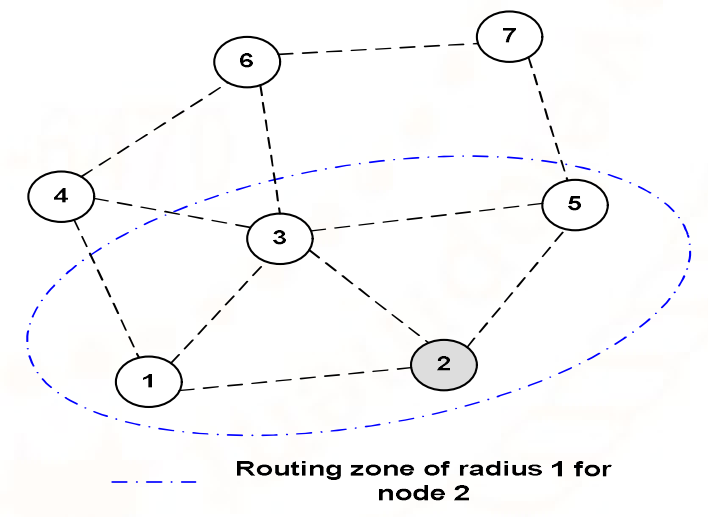

Figure 2.2: Routing zone and zone radius

In order to discover border nodes it uses BRP .if a node successfully inverted the entry in zone through border node so it will first request to the indra-zone nodes regarding routing information and request to the source node. In this process massage has to be broadcast and retransmitted until or unless the related destination is not find, In this way source node will 
get response from many nodes, so that source node select the best rout, designed a metric and computer the best route for delivery.

\section{RRS ALGORITHM}

\section{Module 1: Making P2P (Ad hoc) network model:}

New Algorithm 1: coverage and position based selfadaptive quick node deployment algorithm

In this algorithm we develop a new prototype/algorithm to make/place/move the nodes in the network area.

Consideration for this algorithm:

- Total network area $\mathrm{x}$ and $\mathrm{y}$ axis length

- Total number of nodes

- Number of movable nodes.

- Number of fixed nodes.

- Transmission range/Coverage area for each node.

- Total Simulation time.

- Speed of the simulation.

- Energy level of each node.

Steps to implement this algorithm:

- Find the total network area. Say for an example 1000x1000 (xy length)

- Place all the nodes in $(0,0)$ position. Say for example total nodes are 50 . So 50 nodes will be placed in the position $(0,0)$

- Find each nodes coverage area. Say for an example the coverage area will be $250 \mathrm{~m}$ for each node

- Find Energy level for each node. Say for an example an energy level is $100 \mathrm{~J}$ for each node

- Place the fixed nodes: Say for example 10 nodes are fixed means, we could place those fixed nodes in an equal distance distributed manner in the total network area with even exceeding the coverage area. Exceeding the coverage area means the fixed nodes are cannot be able to communicate directly. It needs some intermediate nodes (movable nodes) to pass the data.
Place the moveable nodes: Say for example 40 nodes are moveable nodes; we need to follow below steps to place the moveable nodes in the network.

- Find the distance between the static nodes.

- Find the coverage length.

- Find how many nodes can be placed in between the fixed nodes to communicate between fixed nodes.

- Calculate the energy level and give the balance load when placing moveable nodes in between fixed nodes.

- Make sure that all the nodes has at least one neighbor node to transfer the packets. Means any of one node should be there in to the coverage area for all the nodes to send and receive packets/data.

Provide movement to the moveable nodes: Say for example 40 nodes is moveable; all should be in moving state. Follow the below steps to make movement of all the moveable nodes.

- Find the distance between all the nodes.

- Find the energy level dynamically when simulation at run time.

- Find total time of the simulation and calculate the energy level at run time

- Make sure if any node don't have any neighbor, move the next nearer node into the coverage area to avoid link failure

- Based on speed of the network, need to manage the energy level to avoid the zero level energy at any time

- If any moveable node having less/low energy, make it as fixed node.

- Always link should be there in the path where the data is transferring.

\section{Module 2: Design a reliable routing protocol:}

New Algorithm 2: Best resource picker reliable routing protocol (BRPR). We develop a new protocol called Best resource picker reliable routing protocol (BRPR) which should take the minimum hop count, no link failure, fast transfer, high throughput and packet delivery ratio with less energy consumption. 
Steps in the algorithm:

1. Find the source node and destination node.

2. Find how many source and destination nodes are in the network.

3. Based on number of source and destination, we need to provide the load balancing for the routing process

4. Send Request from destination to source with minimum number of hop .

5. Once source, got the request from the destination, the below process will get started.

6. Make the empty routing table.

7. Find the neighbor nodes list from the source node

8. Select the particular node which is available in routing table with high priority.

9. Using the above formula, fill up the routing table from source to destination to pick up the intermediate nodes.

10. Once the best path formed, Pre calculation to check the path won't get break till the end of the simulation.

New Algorithm 3: Pre-calculation step includes the process of node traverse direction, coverage area, energy level, linkage between nodes till the end of the simulation.

11. Based on the pre-calculation result, we do the bandwidth adjustment algorithm to avoid the link failure.

12. New Algorithm 4: Bandwidth adjustment algorithm will increase the bandwidth of the link which are in active and remaining node links bandwidth will get decrease since it is not participating in the transmission.

13. When it conforms it is strong path, Start sending the packets.

14. Do the same steps till the end of the simulation since it is ad hoc network.

\section{SIMULATION RESULT}

Figure 4.1 shows that the delay in various protocols. Delay state that the delays in transfer of data till the destination in terms of milliseconds. Compared with other protocol, the proposed protocol RRS transfers the packet without any delay till the destination resource.

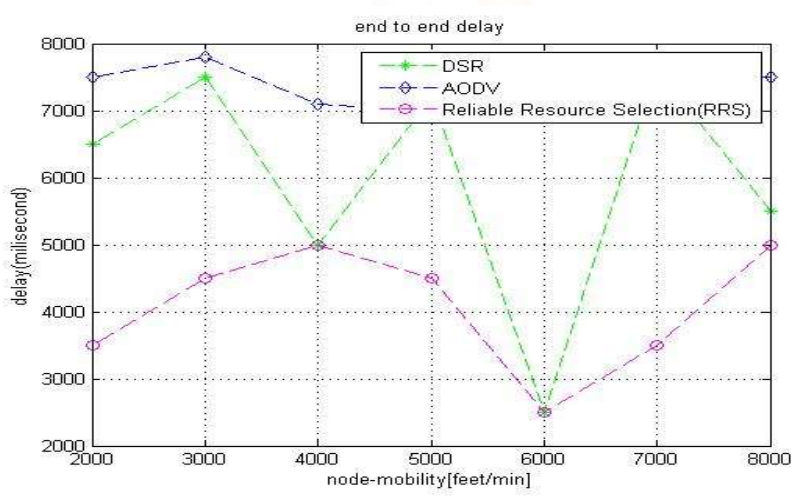

Figure 4.1 Delay Vs node mobility comparison for different protocol

Figure 4.1 explains that the throughput value of RRS protocol. Throughput explains the speed of transfer of data till the destination resource. Compared with other protocol like- AODV protocol and DSR protocol, RRS protocol transfers the packet in very high speed.

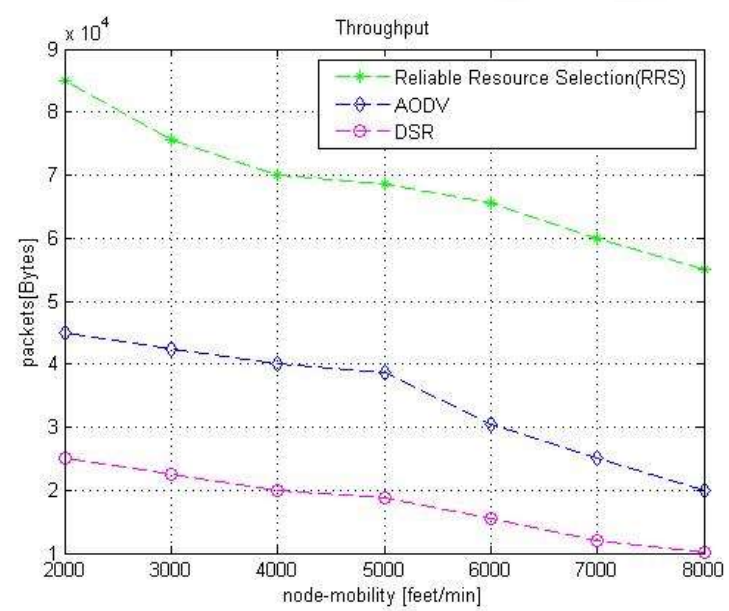

Figure 4.2: Throughput Vs node mobility comparison for different protocol

Figure 4.3 explains the packet delivery ratio of different protocols. Packet delivery ratio delivers the yields packets to the destination resource. It is expressed in terms of percentage. The proposed RRS protocol delivers the packet in the best manner with 
high ratio to avoid the loss of packet compared with other protocols.

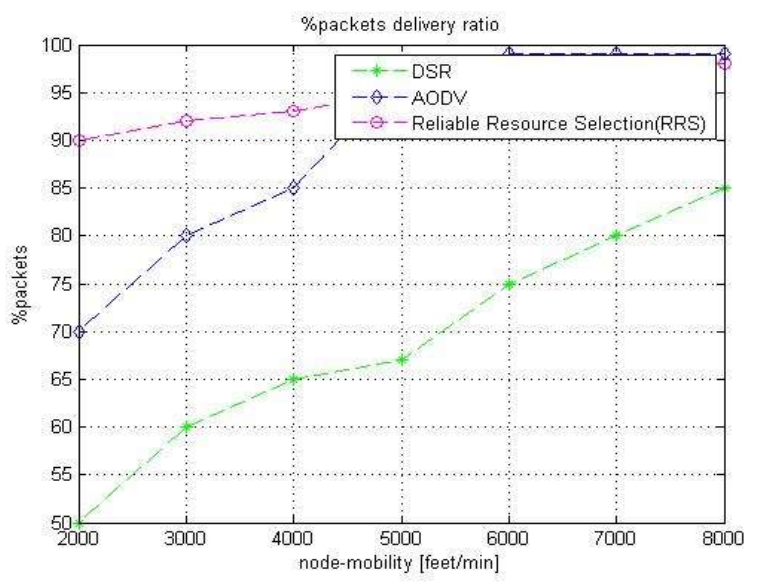

Figure 4.3: \%packets delivery Vs node mobility comparison for different protocol

\section{CONCLUSION}

This paper mainly focus on the services provided by traditional routing protocols used by $\mathrm{P} 2 \mathrm{P}$ distributed network, the objective was to identify the problems issues of different routing scheme and their isolation with more suitable algorithm to improve the pitfalls which we identified and now to proposed a new routing model called RRS to improve performance efficiency compare to the table driven and on demand based routing strategy. In related work session we discussed many protocols of different category along with their initial working details, so that one can design a new model that resolves the issues of traditional routing strategy.

The proposed protocol RRS transfer the data from the source resource, to the destination resource in high speed, high packet delivery ratio and without any delay with the consideration of resources distance, angle, bandwidth and energy level. Finally, we conclude that, if we follow the step by step implementation of RRS protocol in network, get a better performance in transferring data and $\mathrm{p} 2 \mathrm{P}$ network structure.

\section{REFERENCES:}

[1] Amit Mangalekar, SuhasMudgal,SarjeraoMasal, "Energy Efficient Routing Protocol in MANET Using NS-2", International Journal Of Core Engineering \& Management (IJCEM) Volume 1, Issue 10, pp.163-175 January 2015.
[2] Rüdiger Schollmeier, A Definition of PeertoPeer Networking for the Classification of PeertoPeer Architectures and Applications, Proceedings of the First International Conference on PeertoPeer Computing, IEEE (2002).

[3] J. Broch et al, "A Performance Comparison of Multi-Hop Wireless Ad-hoc Network Routing Protocols," Proceedings of the MOBICOM'98, pp. 85-97, October 1998.1

[4] J.E.M. Royer and C.-K. Toh, "A Review of Current Routing Protocols for Ad-Hoc Mobile Wireless Networks," IEEE Personal Communications, pp. 46-55, April 1999.

[5] C. Siva Ram Murthy, B.S. Manoj, "Ad Hoc Wireless Networks: Architectures and Protocols", Prentice Hall Publishers, May 2004, ISBN 013147023X.

[6] C.-K. Toh, “Ad Hoc Mobile Wireless Networks: Protocols and Systems", Prentice Hall publishers, December 2001, ISBN 0130078174.

[7] E.M. Royer and C.-K. Toh, "A Review of Current Routing Protocols for Ad-Hoc Mobile Wireless Networks," IEEE Personal Communications, pp. 46-55, April 1999.

[8] M. K. Gulati and K. Kumar, "QoS routing protocols for mobile ad hoc networks: a survey," International Journal of Wireless and Mobile Computing, vol. 5, no. 2, pp. 107-118, 2012. http://dx.doi.org/10.1504/IJWMC.2012.046783

[9] Min Zhu, Dengyin Zhang, Zhanxiang Ye, Xuemei Wangand Jin Wang "A Network Coding Based Routing Protocol in Wireless Sensor Networks"I nternational Journal of Future Generation Communication and Networking. Vol. 8, No. 2 (2015), pp. 365-372.

[10] S.Yu, B. Zhangand C. Li, "Routing protocols for wireless sensor networks with mobile sinks", a survey, IEEE Communications Magazine,vol.52,no.7, 2014.

[11] HajerBargaoui, Nader Mbarek, Olivier Togni, MounirFrikhaInternational Journal on Advances in Networks and Services, vol 8 no 1 \& 2, year 2015 pp 27-41.

[12]Ronal Benitto Dhas .A1, Ruby.D2,"A QOS Based Routing in Mobile Ad-Hoc Networks" 
International Journal of Advanced Research in Computer and Communication Engineering Vol. 4, Issue 5, May 2015, pp-551-556.

[13] Ali Mohamed E. ,’Neighbor-Based Dynamic Connectivity Factor Routing Protocol for Mobile Ad Hoc Network" 2016, Volume: 4 Pages: 8053 8064, DOI:10.1109/ACCESS.2016.2623238.

[14] Raphael Ernst,"Reducing MANET neighborhood discovery overhead "39th Annual IEEE Conference on Local Computer Networks, $2014 \quad$ Pages: $374 \quad$ - 377 , DOI:10.1109/LCN.2014.6925795.

[15] Maximiliano Andres Eschoyez,"Mobile Multihop Ad Hoc Networks: Simulation and Study IEEE Latin America Transactions, 2007, Volume: 5, Issue: 1 ,Pages: 21 - 27, DOI: 10.1109/T-LA.2007.4444528 IEEE Journals \& Magazines.

[16] Munir A., Qazi I.A., On Achieving Low Latency at Data Centers 2013. LCN 2013. IEEE Transaction on Networking Technology.
[17] Carvahol, T.Kim, H.S. Neves,N. PACE your Network: Fair and Controllable Multi Tenant Data Center Network, IEEE J. Sel. Areas Commun., vol. 24, February 2013.

[18] Estevez C., Angulo S. A Carrier Ethernet Oriented Transport Protocol with a Novel p2p and QoS integration IEEE Proceedings for Computer Network and Mobile Computing, 2012, pp. 45-50.

[19] Estevez C.,Angulo S. P2P services with the integration of QoS Service architecture , IEEE Proceedings for Computer Network and Mobile Computing, 2012.

[20] Mingwei Li, Yuanwei Jing, Feedback based P2P Protocol for Wireless Sensor Networks -Control and Decision (CCDC,),2012, IEEE Conference. 